\title{
Herausforderungen sind Lebens-Chancen
}

\section{Nicole Furgler}

Generalsekretärin ad interim

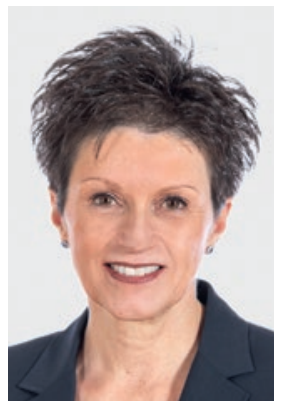

Herausforderungen?! Ja, an diesen hat es im zu Ende gehenden Jahr wahrlich nicht gefehlt! Die ganze Welt ist weiterhin durch Corona gefordert, wie es Ursina Pally Hofmann bereits im letztjährigen DezemberEditorial treffend beschrieben hat. Dahinter verschwindet derzeit beinahe die Bedeutung der Klimaproblematik, obwohl sie längerfristig wohl als grösseres Problem Bestand haben wird. Trockenheit, Grossfeuer und Überschwemmungen sowie die damit verbundenen Flüchtlingsströme rufen uns dies unerbittlich immer wieder in Erinnerung.

In dieser herausfordernden Zeit zählen oder hoffen wir zumindest auf Solidarität, Verständnis, Konsens und Unterstützung. Zu unserem Erstaunen tritt jedoch etwas ganz anderes zutage: Es kommen trennende, spaltende, widerständige Tendenzen und gar Gewalt zum Vorschein. Das sind wir uns zumindest in der Schweiz bisher nicht gewohnt. Auch der Gesundheitsbereich bleibt von dieser Entwicklung nicht verschont; die Gesundheitspolitiker, die Spitäler und Arztpraxen, die Pflegenden und Ärztinnen sind davon betroffen.

Das Ausmass und die Intensität dieser Herausforderungen können uns lähmen, überwältigen und ein Gefühl der Ohnmacht und Einsamkeit aufsteigen lassen. Umso wichtiger scheint es mir, dass wir in dieser Situation zusammen nach Lösungen suchen, nach einem Weg nach vorne und dass wir diesen auch ge-

\section{Vielfältige Herausforderungen können uns lähmen, sie bieten aber auch die Chance der Weiterentwicklung und des Begehens neuer Wege.}

meinsam gehen. Unsere Projekte, Gremien, Versammlungen, Sitzungen und Gespräche bieten uns dafür täglich Gelegenheiten. Sie können uns aus der Machtlosigkeit oder Vereinzelung herausholen, auch und gerade, wenn es dabei angeregte Auseinandersetzungen, manchmal wohl auch Ärger gibt und die Findungsprozesse weder einfach noch kurz sind. Aber so wenden wir uns einander zu, begegnen einander - auch wenn es derzeit oft virtuell ist -, bleiben wir in intensivem Kontakt miteinander, mit der Welt und mit unserem eigenen Innersten: Wir bleiben lebendig und in Bewegung.

In Bewegung, das war auch das zutreffende Bild für die Situation im Generalsekretariat der FMH in diesem Jahr. Der Wechsel im Präsidium der FMH und auch diverse personelle Wechsel und Vakanzen an Schlüsselpositionen im Generalsekretariat halten uns mächtig auf Trab. Die altbekannte und vertraute physische Sitzung ist dem virtuellen Zusammentreffen gewi-

Ich freue mich auf das Neue im kommenden Jahr und bin dankbar für das, was wir gemeinsam bisher bereits geschafft haben.

chen. Eine virtuelle Sitzung jagt die nächste. Die Anforderungen an die Beschäftigten wandeln sich in einem immer höheren Tempo - die Ansage lautet, flexibel und anpassungsfähig zu bleiben. Wie im gesamten Gesundheitswesen gehört es daher auch bei uns im Generalsekretariat zu den drängenden Anliegen, die Arbeitslast für alle in tragbaren Massen zu halten.

Gleich zu Beginn des neuen Jahres wird das Generalsekretariat der FMH wieder in sein neu gestaltetes Gebäude an der Elfenstrasse zurückkehren können. Die offene Büroform ("open space») wird uns die abteilungsübergreifende Zusammenarbeit erleichtern und den offenen Dialog, den einfachen Informationsfluss und den Teamgeist fördern. Diese grosse Veränderung im Büroalltag wird für uns jedoch eine Herausforderung sein, die wir nur im Miteinander erfolgreich bewältigen können.

Ja, unser Leben hat sich verändert und wird sich weiter verändern. Das ist zwar herausfordernd, aber bietet uns die grosse Chance, lebendig und in Bewegung zu bleiben, einander intensiv zu begegnen sowie eine gemeinsame Kultur der Solidarität und der persönlichen Zuwendung entwickeln und pflegen zu können. Auf all das freue ich mich für das kommende Jahr, und ich bin allen dankbar für das, was wir diesbezüglich schon in diesem Jahr erfahren durften. 